\title{
PERIFÍTON E ESTADO TRÓFICO EM DOIS MANANCIAIS DE ABASTECIMENTO EM ARARAQUARA (SP)
}

\author{
COSTA-MENDONÇA, G. ${ }^{1 *}$; MENDONÇA NETO, N.A. ${ }^{2}$; COSTA, R.C.A. ${ }^{1}$; DELLAMANO-OLIVEIRA, \\ M.J. ${ }^{3} \&$ COLOMBO-CORBI, V. ${ }^{4}$
}

1- Laboratório de Fotointerpretação e Geomática, Departamento de Engenharia Rural, Universidade Estadual Paulista (UNESP), Faculdade de Ciências Agrárias e Veterinárias, Jaboticabal, SP, Brasil.

2- Centro de Estudos de Insetos Sociais, Departamento de Biologia, Universidade Estadual Paulista (UNESP), Instituto de Biociências, Rio Claro, SP, Brasil.

3- Departamento de Botânica, Universidade Federal de São Carlos (UFSCAR), São Carlos, SP, Brasil 4- Programa de Pós-Graduação em Desenvolvimento Regional e Meio Ambiente, Universidade de Araraquara (UNIARA), Araraquara, SP, Brasil.

*Corresponding author: gislaine.cmendonca@gmail.com

\begin{abstract}
Costa-Mendonça, G. ${ }^{*}$; Mendonça Neto, N.A. ${ }^{2}$; Costa, R.C.A. ${ }^{1}$; Dellamano-Oliveira, M.J. ${ }^{3}$ \& Colombo-Corbi, V. ${ }^{4}$ (2018). Perifíton e Estado Trófico em dois Mananciais de Abastecimento em Araraquara (SP). Braz. J. Aquat. Sci. Technol. 22(1). elSSN 1983-9057. DOI: 12911/bjast.v22n1. This study aimed to identify the periphyton community and their responses to abiotic changes in water reservoirs public supply in Araraquara - SP (Ribeirão das Cruzes and Córrego Águas do Paiol). To access these stocks conditions, the phytoplankton presence, genus and species was identified. Also, physical, chemical parameters and total nutrients during the rainy and dry season were monitored in situ. The results demonstrated an increase in phosphorus and total nitrogen during the rainy season, in both sources. It was associated with the hauling of allochthonous material by rainfall. However, the column water nutrients increase was not enough to modify the water quality at the sites, since both reservoirs presented oligotrophic conditions during the analyzed periods. The periphytic community composition reflected the adequate water conditions in both reservoirs, with Zygnematophyceae species predominance. This is a good predictor of oligotrophic environments and low pollution load. However, the work emphasized the importance of vegetation cover recovery in studied spring's microbacia as a conservationist measure, since urban and agricultural areas are expanding in the municipality.
\end{abstract}

Key Words: Periphyton, environmental monitoring, public supply.

\section{INTRODUÇÃO}

A distribuição irregular e a alta demanda por água intensificaram, durante anos, o armazenamento e a construção de reservatórios destinados ao abastecimento público. Esses, muitas vezes sob uma gestão ineficiente e influência das ações antrópicas, sofrem com o desequilíbrio da quantidade e qualidade dos recursos hídricos disponíveis (Amorim, 2016). Os Reservatórios de água são ambientes artificiais, cuja dinâmica e funcionamento podem ser influenciados pelo clima, aspectos hidrológicos e morfométricos (Espíndola et al., 2000; Straskraba \& Tundisi, 2000).

Apesar de serem extremamente importantes, estes sistemas frequentemente recebem grande aporte de poluentes e de diversos contaminantes, oriundos das atividades humanas, ocasionando sua degradação e eutrofização (Cunha et al., 2010). Consequentemente, ocorre alteração da estrutura das comunidades aquáticas, com incidência de florações de microalgas e cianobactérias (Buratti et al., 2017), que podem afetar a coloração, o odor e o sabor da água de abastecimento (Dzialowski et al., 2009; Sun et al., 2013; Olsen et al., 2016). Além disso, essas florações podem representar grande perigo para o consumo humano, devido à capacidade que diversas espécies apresentam de produzirem toxinas (Calijuri et al., 2006; Graham et al., 2010).

Neste contexto, o monitoramento desses ecossistemas é fundamental para a avaliação das condições da água, além de embasar medidas preventivas e restauradoras. A avaliação do grau de trofia e/ou qualidade da água de um curso hídrico é uma importante ferramenta e deve ser realizada através do acompanhamento dos parâmetros físicos, químicos e biológicos (Tundisi \& Tundisi, 2008; Lobo, 2013). O acompanhamento dos parâmetros biológicos vem apresentando resultados satisfatórios na investigação do estado e da integridade dos ecossistemas aquáticos brasileiros (Tundisi, 2003; Arias et al., 2007). O uso de bioindicadores possibilita a avaliação momentânea do estado ecológico destes ambientes, através da utilização de organismos sensíveis às mudanças, que indicam a presença de contaminantes ou alterações no ecossistema (Garcia et al., 2017).

Dentre os inúmeros organismos a serem empregados na classificação ecológica dos ecossistemas aquáticos, as microalgas (fitoplâncton ou 
perifíton) constituem-se importantes bioindicadores, pois refletem os impactos antrópicos. As microalgas incluem espécies tolerantes e espécies sensíveis à poluição (Bellinger \& Sigee, 2010). Importantes no processo de produção primária nos ambientes aquáticos, as microalgas atuam diretamente nos processos de autodepuração dos cursos d'água, pela absorção dos nutrientes dissolvidos na água. A presença de determinadas espécies permite aferir o grau de recuperação do ambiente (Masseret et al., 2000).

As microalgas têm sido amplamente utilizadas como bioindicadores da qualidade da água, pois fornecem informações sobre o comportamento temporário de poluentes e, da mesma forma, permitem a avaliação de informações ou mudanças ambientais de curto prazo (Garcia et al., 2017). As comunidades algais, tanto perifíticas quanto planctônicas, possuem atributos importantes para bioindicação, pois se disseminam em praticamente todos ambientes aquáticos e são comumente ricas em espécies (Vadeboncoeur. \& Steinman, 2002).

No entanto, o modo de vida séssil confere ao perifíton vantagens sobre o fitoplâncton. Portanto, estas microalgas não migram sob condições adversas, constituindo uma comunidade espacialmente compactada, que responde prontamente às mudanças ambientais. Desta forma, as alterações do ambiente geram respostas rápidas na estrutura do perifíton quando comparados com outros organismos (Mccormick \& Stevenson, 1998; Newman et al., 2003). As microalgas perifíticas podem indicar também condições de eutrofização do ambiente em função da elevada sensibilidade de algumas espécies (Mccormick \& Stevenson, 1998; Dodds, 2003); ou da capacidade de acumulação de nutrientes e poluentes (inseticidas, herbicidas e fungicidas, metais pesados e materiais orgânicos) de outras microalgas (Sládecková,1991; Li et al., 2010).

Por outro lado, o desenvolvimento acelerado de microalgas perifíticas pode originar formas planctônicas (bloom), que causam prejuízos nos sistemas de captação e tratamento de água, como problemas na vedação de filtros, incrustações em caixas d'água e corrosão de comportas (Sladecková, 1991; Bicudo et al., 1995). Ocorre, portanto, a necessidade de se monitorar a água dos reservatórios, uma vez que alguns fatores abióticos podem favorecer o crescimento das algas perifíticas. Como por exemplo, a disponibilidade de nutrientes, que é um dos principais fatores controladores do crescimento das microalgas (Vercellino \& Bicudo 2006; Ferragut \& Bicudo, 2012).

Desta forma, este estudo tem como principal objetivo identificar a comunidade de microalgas perifíticas e sua resposta às condições limnológicas dos principais reservatórios de água para abastecimento público no município de Araraquara (Ribeirão das Cruzes, Córrego Águas do Paiol), como ferramenta para a avaliação da qualidade da água e do grau de trofia desses reservatórios.

\section{MATERIAIS E MÉTODOS}

\section{Área de Estudo}

A área de estudo compreende dois afluentes da bacia hidrográfica do Rio Jacaré - Guaçu, que integram a Unidade de Planejamento e Gestão dos Recursos Hídricos (UPGRH - 13) da bacia do TietêJacaré (Tundisi et al., 2008): o Ribeirão das Cruzes e o Córrego Águas do Paiol (Figura 1). O Ribeirão das Cruzes constitui um dos principais afluentes da bacia hidrográfica do rio Jacaré-Guaçu, localizado a noroeste da cidade, possui uma sub-bacia de $173 \mathrm{~km}^{2}$ dentro do perímetro urbano e recebe a contribuição do Córrego Águas do Paiol entre outros.

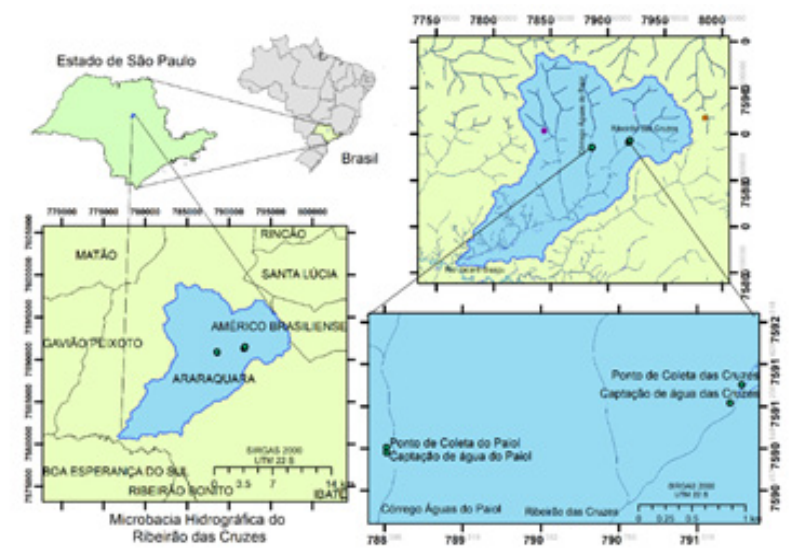

Figura 1 - Localização do Ribeirão das Cruzes e Córrego Águas do Paiol no município de Araraquara (SP).

Os dois mananciais estudados são destinados à captação e tratamento de água para abastecimento público no município de Araraquara, SP (214ㄱ'40"S, 48 10'32"W). Segundo o Departamento Autônomo de Água e Esgoto do Município de Araraquara (DAAE), juntas, as duas represas são responsáveis por aproximadamente $50 \%$ do abastecimento do município.

Os solos da região de Araraquara são ocupados por campo (especialmente cerrado) e agricultura, com destaque para a cultura de cana-de-açúcar, que ocupa áreas extensas próximas às margens dos mananciais. O clima do município de Araraquara é do tipo Aw segundo a classificação de Köppen, tropical de verão chuvoso e inverno seco, com precipitação média anual $1352 \mathrm{~mm}$ e temperatura média variando de $19,1^{\circ} \mathrm{C}$ no mês mais frio, a $24,8^{\circ} \mathrm{C}$ no mês mais quente. 


\section{Planejamento amostral}

O estudo foi conduzido no Ribeirão das Cruzes e no Córrego Águas do Paiol, com uma coleta em fevereiro e outra em março, abrangendo o período de chuva. A coleta de julho e a de agosto representaram o período de seca. As coletas de água e de material perifítico foram realizadas o mais próximo possível dos sistemas de captação de água nos dois mananciais. No Ribeirão das Cruzes, as coletas se deram a montante da represa de captação, e no Córrego Águas do Paiol, a jusante devido ao difícil acesso ao ponto anterior da captação (Figura 1).

\section{Variáveis ambientais e limnológicas}

Os dados de temperatura do ar e precipitação média mensal do período estudado foram obtidos a partir do banco de dados do Instituto Nacional de Meteorologia (INMET) e do Departamento de Águas e Energia Elétrica (DAEE), respectivamente. As medidas dos parâmetros físicos e químicos da água constituíram-se dos registros "in loco" do teor de oxigênio dissolvido na água (OD), temperatura (Temp.) e pH. Essas medidas foram realizadas no período da manhã, entre 8:00 e 11:00 horas, com o aparelho multisensor da marca Yellow Springs, modelo 556.

As coletas de água para análise de nutrientes (nitrogênio e fósforo total) foram realizadas com garrafa de Niskin (General Oceanics), previamente limpa, desinfetada e enxaguada com água do reservatório. As amostras de água foram armazenadas em frascos de polietileno esterilizados ( $2 L$ ) e mantidas em freezer até a realização das análises. A determinação de nitrogênio total (NT) e fósforo total (PT) foram realizadas de acordo com as técnicas descritas por Valderrama (1981).

\section{Índice de estado trófico}

O IET dos dois reservatórios foi determinado de acordo com Lamparelli (2004). O cálculo utiliza como variável independente a concentração de fósforo total (PT) dado em $\left(\mu \mathrm{g} \cdot \mathrm{L}^{-1}\right)$.

\section{Comunidade perifítica algal}

O perifíton foi amostrado através de coletas randômicas dos pecíolos, folhas e raízes das macrófitas distribuídas no ponto amostral, compreendendo a área de $245 \mathrm{~m}^{2}$ e perímetro de $67,2 \mathrm{~m}$ no Ribeirão das Cruzes e, uma área de 99,3 $\mathrm{m}^{2}$ e perímetro de $57,8 \mathrm{~m}$ no Córrego águas do Paiol por intermédio de amostrador de área conhecida. Este material foi acondicionado em sacos plásticos estéreis e levado ao laboratório para remoção do material ficoperifítico. O perifíton foi removido do substrato por meio de raspagem com o auxílio de pincel, estilete, escovas de cerdas e jatos de água destilada. O material resultante da separação do perifíton e substrato foi acondicionado em frascos de vidro e fixado com solução de formalina $4 \%$ (Bicudo e Menezes 2006). A análise da composição florística compreendeu a identificação dos táxons em microscópio óptico Zeiss - Axioscop, com resolução máxima de 2560 vezes. A identificação dos táxons se deu ao menor nível possível, utilizando-se as referências de Bourrelly (1981), Komárek \& Fott (1983), Bourrelly (1985), Anagnostidis \& Komárek (1988), Komárek \& Anagnostidis (1989), Bicudo e Menezes (2006); Franceschini et al. (2010).

\section{Análise de dados}

As médias e os desvios padrões das variáveis limnológicas foram determinados para os pontos de coleta e períodos. Para a verificação da normalidade e homocedasticidade dos dados, foram aplicados os testes de Lillierfors e Bartlett, assim como a análise residual ao nível de $5 \%$ de significância $(p=0,05)$ (Siegel,1975). Para a verificação da variação espacial e sazonal dos ambientes em relação às variáveis limnológicas, foi realizada a análise de componentes principais (PCA) de acordo com o critério de Kaiser (1958), sendo previamente padronizada a matriz dos dados limnológicos [(Xij-Xi) / Si] (Legendre \& Legendre, 1998). A análise de variância (ANOVA) oneway, foi aplicada às pontuações geradas pelo PCA, testando as diferenças das variáveis limnológicas entre os ambientes e períodos estudados(Siegel,1975). Quando as diferenças significativas foram detectadas, o Teste de Tukey foi utilizado para comparações post hoc. Todas as análises foram realizadas através do programa STATISTICA versão 7.0.

\section{RESULTADOS}

\section{A Caracterização ambiental e limnológica}

Ambos os mananciais passam por áreas urbanas e rurais. No ponto de coleta do Ribeirão das Cruzes (à montante da represa de captação), foi observada a presença de mata ciliar; enquanto que no ponto de coleta do Córrego Águas do Paiol (à jusante da represa de captação), ocorreu forte influência antrópica, devido à pastagem e presença de animais no entorno, além de residências próximas ao leito do córrego.

A temperatura do ar e a precipitação média mensal do município foram superiores durante o período chuvoso em relação ao período de seca. As temperaturas médias nos meses de fevereiro e março variaram de $22^{\circ} \mathrm{C}$ a $23^{\circ} \mathrm{C}$, enquanto a precipitação média em ambos os meses variou de $170 \mathrm{~mm}$ a 179 $\mathrm{mm}$. No período de seca, no mês de julho, foram registradas temperatura e precipitação média de $17^{\circ} \mathrm{Ce}$ $18,70 \mathrm{~mm}$, respectivamente. Em agosto, estes valores 
médios foram de $18^{\circ} \mathrm{C}$ e $15,60 \mathrm{~mm}$, respectivamente. Os resultados da análise de componentes principais representaram $98,38 \%$ da variabilidade total dos dados (Figura 2). O CP 1 reteve $79,31 \%$ das informações relevantes, estando negativamente relacionado às maiores concentrações de fósforo e nitrogênio total, maior pluviosidade e temperatura da água e do ar; responsáveis pelo distanciamento dos pontos de coleta em ambos os mananciais durante o período chuvoso. Neste mesmo eixo, foram positivamente ordenados os pontos de coleta do Ribeirão das Cruzes e Córrego Águas do Paiol relacionados as menores concentrações de nutrientes, precipitação e temperatura, agrupando os dois pontos durante o período de estiagem.

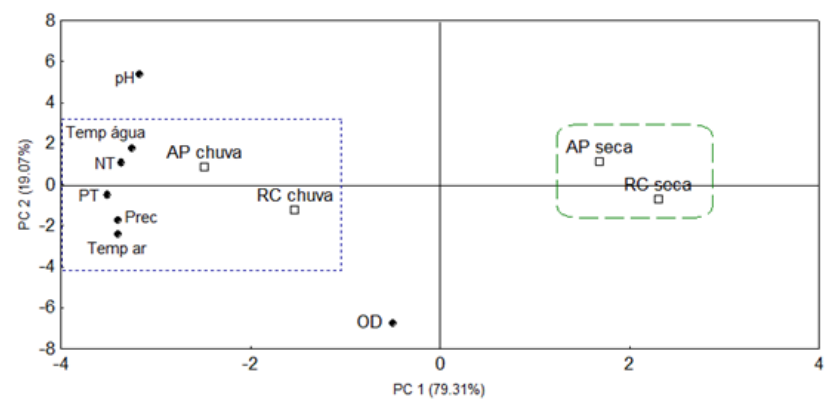

Figura 2 - Interpolação dos autovalores das variáveis limnológicas, precipitação e temperatura nos pontos de amostragem. Onde: Ponto de coleta do Ribeirão das Cruzes (RC); ponto de coleta do Córrego Águas do Paiol (AP); período de chuva (chuva); período de seca (seca); quadrado aberto = pontos amostrais; círculos fechados $=$ variáveis.

O segundo eixo, CP 2 explicou $19,07 \%$ dos dados, correlacionado negativamente ao maior teor de oxigênio dissolvido, associado ao Ribeirão das Cruzes durante o período chuvoso. Esta análise apontou a variação da qualidade da água em relação aos períodos de chuva e seca, sem maiores diferenças entre os dois mananciais. A análise de variância confirmou as diferenças entre os dois períodos monitorados (Tabela 1).

Tabela 1- Concentrações médias e desvio padrão das variáveis limnológicas,teste de ANOVA (one-way) dos pontos amostrais e períodos estudados.

\begin{tabular}{lcccc}
\hline \multirow{2}{*}{$\begin{array}{c}\text { Parâmetros } \\
\text { Limnológicos }\end{array}$} & \multicolumn{2}{c}{ Chuva } & \multicolumn{2}{c}{ Seca } \\
\cline { 2 - 5 } & $\mathbf{R C}$ & $\mathbf{A P}$ & $\mathbf{R C}$ & $\mathbf{A P}$ \\
\hline $\mathrm{pH}$ & $6,5 \pm 0,2^{\text {ns }}$ & $6,7 \pm 0,1^{\text {ns }}$ & $6,4 \pm 0,2^{\text {ns }}$ & $6,5 \pm 0,1^{\text {ns }}$ \\
OD (mg. L-1 $)$ & $8,9 \pm 0,1^{\mathrm{a}}$ & $8,2 \pm 0,1^{\mathrm{c}}$ & $8,6 \pm 0,1^{\mathrm{b}}$ & $7,8 \pm 0,1^{\mathrm{d}}$ \\
Temp. $\left(C^{\circ}\right)$ & $21,0 \pm 1,0^{\mathrm{a}}$ & $22,6 \pm 1,2^{\mathrm{a}}$ & $18,7 \pm 0,6^{\mathrm{b}}$ & $19,0 \pm 1,0^{\mathrm{b}}$ \\
Nitrogênio Total $\left(\mu \mathrm{g} \mathrm{L}^{-1}\right)$ & $591 \pm 9,5^{\mathrm{b}}$ & $631 \pm 7.3^{\mathrm{a}}$ & $185 \pm 3,5^{\mathrm{c}}$ & $348 \pm 5,5^{\mathrm{d}}$ \\
Fósforo Total $\left(\mu \mathrm{g} \cdot \mathrm{L}^{-1}\right)$ & $60,9 \pm 3,5^{\mathrm{a}}$ & $64,5 \pm 1,7^{\mathrm{a}}$ & $36,7 \pm 1,0^{\mathrm{b}}$ & $40,85 \pm 4,1^{\mathrm{b}}$ \\
\hline
\end{tabular}

Valores seguidos pelas mesmas letras em cada coluna não diferem significativamente $(\mathrm{p}<0,05) ;>\mathrm{b}>\ldots$... ns= não significativo; - = não avaliado. $\mathrm{RC}=$ Ribeirão das Cruzes; $A \mathrm{P}=$ Córrego Águas do Paiol.
A temperatura da água entre os dois mananciais apresentou pouca variação; no entanto, foi significativamente superior no período de chuva $(p<0,05)$. No Ribeirão das Cruzes, a temperatura média da água oscilou entre $18^{\circ} \mathrm{C}$ no período de seca e $22^{\circ} \mathrm{C}$ no período chuvoso; enquanto que no córrego Águas do Paiol, variou entre $19{ }^{\circ} \mathrm{C}$ e $24^{\circ} \mathrm{C}$, respectivamente (Tabela 1).

Os teores de oxigênio dissolvido estiveram acima de $7 \mathrm{mg} / \mathrm{L}$, com águas mais oxigenadas durante o período chuvoso no Ribeirão das Cruzes. No córrego Águas do Paiol foi encontrado o menor valor médio de oxigênio dissolvido no período de seca (Tabela 1). Em geral, o pH da água não apresentou variação significativa $(p>0,05)$ entre os dois mananciais ou entre ambos períodos estudados, com águas levemente ácidas nos dois períodos estudados, oscilando de 6,3 a 6,8 (Tabela 1).

Maiores concentrações de nitrogênio total foram detectadas no período chuvoso em ambos os mananciais. No entanto, no córrego Águas do Paiol foram observadas concentrações superiores de nitrogênio total, variando de $348 \mu \mathrm{g}$. L-1a $631 \mu \mathrm{g}$. $\mathrm{L}^{-1}$; enquanto no Ribeirão das Cruzes, oscilou de185 $\mu \mathrm{g} . \mathrm{L}^{-1}$ a $591 \mu \mathrm{g}$. L-1. Padrão similar foi verificado nas concentrações de fósforo total, superiores no período de chuva em ambos os mananciais, variando de $41 \mu \mathrm{g}$. $\mathrm{L}^{-1}$ a $65 \mu \mathrm{g}$. $\mathrm{L}^{-1}$ no córrego Águas do Paiol, e de $36 \mu \mathrm{g}$. $\mathrm{L}^{-1}$ a $60 \mu \mathrm{g}$. $\mathrm{L}^{-1}$ no Ribeirão das Cruzes (Tabela 1). Com base nas concentrações de fósforo total, os resultados de IET para ambos os mananciais variaram de 48 a 51 , indicando que estes ecossistemas apresentaram características oligotróficas durante todo o período estudado.

\section{Comunidade perifítica algal}

Foram identificados um total 16 taxa de algas perifíticas, distribuídos nas seguintes classes (Tabela 2): Zygnematophyceae, Bacillariophyceae e Cyanobacteria (Tabela 2). No córrego Águas do Paiol foram registrados 8 táxons, composto por Zygnematophyceae $(50,00 \%)$, Bacillariophyceae $(37,5 \%)$ e Cyanobacteria (12,5\%). Já no Ribeirão das Cruzes foram registrados 7 táxons, representados por Zygnematophyceae $(42,8 \%)$, Bacillariophyceae $(28,65$ $\%)$ e Cyanobacteria (28,65 \%) (Figura 2). Dentre as espécies identificadas, Spirogyra sp. foi o único táxon encontrado em ambos os reservatórios, totalizando as 16 espécies encontradas. 
Tabela 2 - Composição e ocorrência específica nos pontos e períodos amostrados.

\begin{tabular}{lcccc}
\hline \multirow{2}{*}{ TAXA } & \multicolumn{2}{c}{ Chuva } & \multicolumn{2}{c}{ Seca } \\
\cline { 2 - 5 } & RC & AP & RC & AP \\
\hline BACILLARIOPHYCEAE & & & & \\
Eunotia sp 1 & & & + & \\
Eunotia sp 2 & - & - & - & + \\
Navicula sp 1 & + & - & - & - \\
Navicula sp 2 & - & - & + & - \\
Pinnularia sp & - & - & - & + \\
CYANOPHYCEAE & & & & \\
Aphanocapsa sp & - & + & - & - \\
Cianobactéria NI & + & - & - & - \\
Planktothrixsp. & + & - & - & - \\
ZYGNEMATOPHYCEAE & & & & \\
Closterium moliferum & - & - & + & - \\
Closterium irembergii & - & - & + & - \\
Closterium navicula & - & - & - & + \\
Closterium sp & - & - & - & + \\
Gonatozygon sp. & - & + & - & + \\
Netrium sp. & - & - & - & + \\
Spirogyra sp. & + & + & + & + \\
Zygnema sp. & + & - & + & - \\
\hline (+) & & & &
\end{tabular}

(+) presença, (-) ausência; (NI) Não identificado

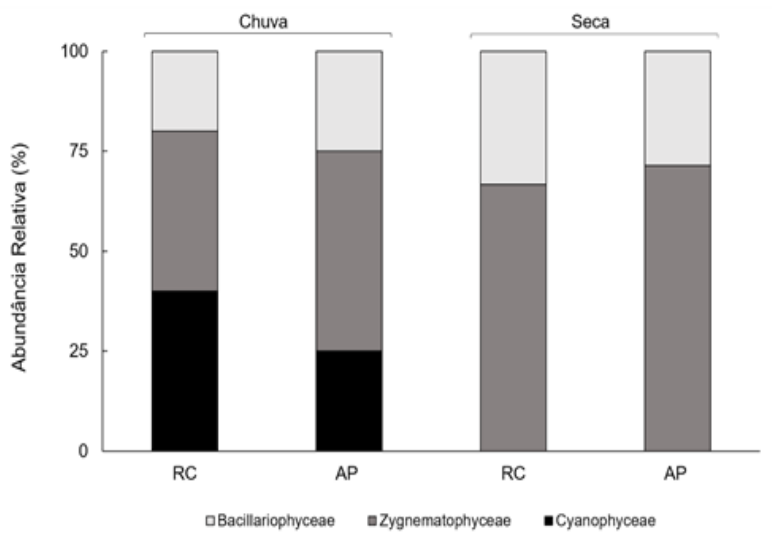

Figura 3 - Abundância relativa (\%) dos grupos de microalgas perifíticas nos pontos e períodos estudados.

Em relação aos períodos estudados, a maior riqueza específica foi apresentada por Zygnematophyceae, seguida por Bacillariophyceae no período chuvoso. As Cyanophyceae ocorreram em ambos os córregos somente no período chuvoso, com maior riqueza de espécies no Ribeirão das Cruzes. A classe Zygnematophyceae apresentou maior riqueza específica e foi predominante nos dois ambientes e nos dois períodos hidrológicos. Essa classe esteve representada, em sua maioria, por indivíduos coloniais, seguidos das formas filamentosas e unicelulares. Dentre as espécies encontradas, destaca-se Spirogyra $\mathrm{sp}$, que ocorreu nos dois córregos nos períodos chuvoso e seco, apresentando aglomerações ao longo das margens do Ribeirão das Cruzes, principalmente no período de estiagem. O gênero Closterium teve a maior diversidade de espécies durante o período de seca.
As demais classes apresentaram menor número de indivíduos. Bacillariophyceae foi representada pelos gêneros Navicula, Eunotia e Pinularia, com apenas uma espécie de Navicula registrada durante o período chuvoso no Ribeirão das Cruzes. A classe Cyanophyceae foi representada, em sua maioria, por talos filamentosos, Planktothrix sp e Cyanobacteria NI predominaram no Ribeirão da Cruzes. A forma colonial Aphanocapsa sp foi a única espécie encontrada no Córrego Águas de Paiol.

\section{DISCUSSÃO}

A temperatura do ar, precipitação e o tipo de cobertura do solo no entorno dos locais monitorados, devem ter contribuído para as condições da água em cada reservatório (Amorim, 2016; Oliveira \& Bicudo, 2017). No período chuvoso, foram registradas águas mais quentes, oxigenadas e com altos teores de fósforo e nitrogênio total em ambos os mananciais, enquanto o $\mathrm{pH}$ da água nos dois pontos foi ligeiramente ácido durante todo o período estudado.

A variação da temperatura da água seguiu os padrões climáticos da região, com valores superiores no verão e temperaturas mais baixas no inverno. A reduzida cobertura vegetal ou a ausência de mata ciliar no entorno dos reservatórios também afetou a temperatura da água, pois conforme o verificado por Krupek et al.(2008), a ausência de cobertura arbórea favorece a incidência dos raios solares na água e, consequentemente, o aumento da temperatura.

$\mathrm{O} \mathrm{pH}$ da água, ligeiramente ácido, refletiu a composição do solo que constitui a bacia hidrográfica, pois na região estudada predominam latossolos vermelhos/amarelos, caracteristicamente ácidos. Além disso, o período chuvoso favorece a diluição das concentrações de cálcio e o aporte de matéria orgânica alóctone, interferindo nos compostos ácidos na coluna d'água (Krupek et al., 2008). No entanto, os níveis de $\mathrm{pH}$ encontrados nos reservatórios são satisfatórios e adequados ao desenvolvimento da vida aquática, segundo os critérios descritos por Vigil (2003).

O uso do solo nas margens dos corpos de água pode ter interferido no perfil de oxigênio das duas represas. Os trechos de mata ciliar no Ribeirão das Cruzes contribuíram para as maiores taxas de OD; enquanto que no córrego Águas do Paiol, o menor nível de oxigênio pode estar associado à presença de pasto e gado no entorno do ponto amostral. Além disso, a ausência de cobertura vegetal nesta área facilitou o carreamento de resíduo orgânico, diminuindo as concentrações de oxigênio dissolvido em função da decomposição de matéria orgânica (Esteves, 2011; Cabral et al., 2012). Entretanto, as concentrações de oxigênio dissolvido nos dois sistemas estão dentro 
dos níveis adequados para água de abastecimento, de acordo com Vigil (2003).

As altas concentrações de fósforo e nitrogênio total, verificadas no período chuvoso, principalmente no córrego Águas do Paiol, devem estar relacionadas à drenagem pluvial das áreas agrícolas e urbanas no entorno desse reservatório. Isso ocorre devido à reduzida cobertura vegetal nas margens dos mananciais, que potencializa a lixiviação de nutrientes para os corpos de água (Oliveira et al., 2010; Cabral et al., 2012). Além disso, as atividades de pecuárias e de plantio facilitam a desagregação do solo pela ação das chuvas e o transporte de sólidos para os reservatórios (Amorim, 2016). Em trabalhos realizados em reservatórios de abastecimento, Freitas et al. (2011) e Oliveira e Bicudo (2017) também verificaram valores mais elevados de nutrientes na estação chuvosa, destacando como fonte significativa de fósforo e nitrogênio os efluentes das industriais de fertilizantes, de alimentos, de laticínios, e de frigoríficos e abatedouros. Embora tenha sido observado um incremento nas concentrações de nutrientes durante o período de chuvas, este aumento não foi suficiente para elevar o grau de trofia desses reservatórios, indicando que a qualidade da água ainda está dentro dos níveis toleráveis ou aceitáveis de eutrofização (Lamparelli, 2004).

$\mathrm{O}$ agrupamento e o distanciamento dos pontos amostrais em relação aos períodos de chuva e de seca, observados na análise de componentes principais, evidenciam a influência sazonal sobre as condições da água; pois no período chuvoso houve forte correlação com as maiores concentrações de nutrientes e com a redução da qualidade da água neste período. A influência da sazonalidade sobre os ecossistemas tropicais se dá pela precipitação, que aumenta o nível da água e das concentrações de nutrientes, alterando o estado hidroquímico e as condições da água dos mananciais (Miretzky et al., 2002; Felisberto \& Rodrigues, 2005).

É interessante citar que os dois córregos em estudo pertencem a classe 4 de acordo com a resolução Conama 357/2005 (CONAMA, 2005).

Quanto à diversidade de microalgas encontrada nos dois ambientes, não foram observadas diferenças significativas, pois ambos apresentaram características limnológicas semelhantes, como águas levemente ácidas e altos valores de nutrientes no período de chuva. Estas características podem ter influenciado a composição das algas perifíticas encontradas nestes ambientes, com a maior disponibilidade de nitrogênio e fósforo (Ferragut \& Bicudo, 2012; Cordeiro et al., 2017). Assim, pode-se inferir que a maior influência na qualidade da água e na composição da comunidade perifítica foi exercida pela hidrologia e pela ação antrópica, pois alterações na química da água, associadas ao processo de urbanização, podem provocar mudanças na estrutura das comunidades de algas perifíticas (Baker et al., 2009; Wu et al., 2009). Além disso, a baixa cobertura de mata ciliar e baixa profundidade dos dois sistemas estudados, potencializou a incidência direta de luz solar que favorece o crescimento das microalgas (Dunck et al., 2013).

Durante o período estudado, a classe com maior representatividade foi Zygnemaphyceae, representada principalmente por desmídias. Isso decorre do tamanho relativamente grande que esse grupo apresenta, e que dificulta a herbivoria; além da grande capacidade de reciclagem de nutrientes (Felisberto et al., 2014). A maior riqueza de táxons se deve principalmente pela facilidade destes organismos em colonizar diversos substratos, mesmo que apenas durante a sua reprodução (Coesel \& Krienitz, 2008; Felisberto et al., 2014). A predominância das desmídias é esperada nestes ambientes, pois as microalgas desta classe crescem no perifíton de reservatórios tropicais, principalmente em águas ligeiramente ácidas e ambientes oligotróficos a mesotróficos (Coesel \& Krienitz, 2008; Bellinger \& Sigee, 2010, Franceschini et al., 2010), como nos sistemas estudados. Aliado a isto, a fraca correnteza verificada nos reservatórios também contribuiu para o estabelecimento destas microalgas (Felisberto \& Rodrigues, 2005; Ngearnpat \& Peerapornpisal, 2007), que podem sinalizar boas condições da água (Coesel, 2001; Št’astný, 2009).

O gênero Closterium apresentou a maior contribuição para a riqueza específica da classe, que pode ter sido favorecida pela presença de macrófitas nos mananciais, já que este táxon de distribuição cosmopolita frequentemente ocorre em ambientes ricos em plantas submersas (Felisberto \& Rodrigues, 2005). Encontrada nos dois reservatórios, Spirogyra sp. possivelmente foi favorecida por sua morfologia, pois os organismos filamentosos são excelentes formas adaptativas e crescem rapidamente em comprimento (Felisberto et al., 2014). As condições da água nos reservatórios contribuíram para a ocorrência da espécie, encontrada frequentemente em ambientes meso a eutróficos, e locais sem corrente ou com baixa velocidade de corrente (Ngearnpat, Peerapornpisal, 2007).

A espécie também apresenta taxas de crescimento relativamente elevadas em ambientes com alta irradiância e temperatura (Berry \& Lembi, 2000), tais como os dois reservatórios estudados. O gênero Spirogyra é cosmopolita, de ocorrência bastante comum em habitats de água doce. Os filamentos desta microalga formam massas mucilaginosas que se aderem ao substrato, ou podem permanecer flutuando na água em pequenos lagos, açudes, córregos e canais ricos em nutrientes (Franceschini et al., 2010). Esse 
comportamento justifica a presença de aglomerados de Spirogyra sp ao longo das margens nos dois reservatórios. Em geral, a diversidade de espécies de Zygnemaphyceae pode ter sido favorecida pelas condições de ambos os mananciais, principalmente sob maiores concentrações de nutrientes e águas lenticulares (Stamenković \& Hanelt, 2017), além dos valores de $\mathrm{pH}$ levemente ácidos e elevada saturação de oxigênio.

A segunda classe de maior representatividade foi Bacillariophyceae, devido às características morfológicas e fisiológicas dessas microalgas, que favorecem o desenvolvimento deste grupo no epífito (Fonseca et al., 2009). De acordo com Murakami et al. (2009), a importância das diatomáceas está associada à sua capacidade de secretar mucilagem e formar pedúnculos ou matrizes mucilaginosas, que permitem a adesão e fixação no substrato. As diatomáceas são colonizadoras rápidas e eficientes, e boa parte de seus representantes apresenta estruturas especializadas de fixação no substrato, como Navicula e Eunotia (Murakami et al., 2009).

As diatomáceas foram encontradas nos dois reservatórios, principalmente no período de seca, possivelmente favorecidas pelas menores concentrações de fósforo e pouca correnteza. Resultados semelhantes foram observados por Winter \& Duthie (2000) e Dunck et al. (2013), os quais registraram maior ocorrência destes gêneros no período de estiagem. Os indivíduos dessa classe são geralmente encontrados em uma ampla variedade de ambientes (Cavati \& Fernandes 2008, Taniwaki et al., 2013); no entanto, Navicula é um indicador de impacto antrópico via despejos industriais (Lobo, 2013; Pandey et al., 2017). Logo, a importância desse gênero no ambiente estudado deve estar associada ao uso das áreas no entorno dos reservatórios com pouca cobertura vegetal, presença de animais e pastagem próximas às margens, principalmente no córrego Águas do Paiol. Já a presença de duas espécies do gênero Eunotia deve estar associada à tolerância que este gênero apresenta às condições de acidez das águas (Wunsam et al., 2002; Andrén \& Jarlman, 2008).

Duas espécies de cianobactérias filamentosas ocorreram no Ribeirão das Cruzes, e uma espécie colonial, no córrego Águas do Paiol, apenas durante o período chuvoso. A ocorrência das cianobactérias neste período foi possivelmente favorecida pelas condições de temperaturas elevadas e aumento nas concentrações de nutrientes nos mananciais (Fernandes et al., 2009). A presença das cianobactérias na estação chuvosa está relacionada com as maiores concentrações de nitrato e fosfato, que ocorrem nesta época (Fonseca e Rodrigues, 2005).

Além disso, Fernandes et al. (2009) relataram que altas temperaturas aceleram o desenvolvimento das cianobactérias, bem como sua capacidade de fixar o nitrogênio atmosférico e estocar internamente o fósforo. Esse comportamento resulta no crescimento destes organismos durante os períodos subsequentes, de deficiência de nutrientes, tornando-as amplamente favorecidas frente aos grupos de microalgas. A predominância de cianobactérias no período chuvoso em reservatórios tropicais tem sido observada por diversos autores (Cavati e Fernandes, 2008, Fernandes et al., 2009; França et al., 2011). Florações de cianobactérias podem causar desequilíbrios, resultantes do acúmulo de matéria orgânica, além de conferirem gosto e odor desagradáveis à água (Dzialowski et al., 2009). A ocorrência de Aphanocapsa sp pode ser um alerta, pois segundo Buratti et al.(2017), esta é uma espécie potencialmente tóxica e, portanto, pode representar risco à saúde humana.

A riqueza e a composição de espécies das algas perifíticas do Ribeirão das Cruzes e do córrego Águas do Paiol estiveram relacionadas ao pH, temperatura da água e disponibilidade de nutrientes, que marcaram os períodos de chuva e seca. De acordo com Bicudo et al. (1995), a temperatura é uma variável controladora do perifíton, uma vez que o processo de sucessão é mais rápido durante os períodos quentes e chuvosos nos diferentes ecossistemas brasileiros (Pellegrini \& Ferragut, 2012). O nitrogênio e o fósforo também são considerados como os principais nutrientes limitantes para o perifíton (Dodds, 2003; Gorman, et al., 2014; Ferragut \& Bicudo, 2012). A ocorrência de cianobactérias no período chuvoso, bem como o predomínio de Zygnemaphyceae e Bacillariophyceae durante os períodos de seca também foram relatados por Pellegrini e Ferragut (2012) no Lago das Ninfeias, reservatório meso tropical em São Paulo (SP).

\section{CONCLUSÃO}

O estudo evidenciou que a condição da água nos dois reservatórios pode ter sido influenciada pela sazonalidade, conforme o padrão climático da região (precipitação e temperatura), e pelas características ambientais, como uso do solo no entorno da microbacia (mata ciliar, áreas de pastagens e ocupação urbana); uma vez que as variações observadas se deram entre os períodos estudados. A intensa pluviosidade em conjunto com a reduzida ou ausente cobertura vegetal elevaram o aporte de nutrientes em decorrência da lixiviação do solo e carreamento de material para dentro dos corpos de água.

A composição específica das algas perifíticas também pode ter sido afetada pelos períodos hidrológicos, embora não tenha havido variação significativa entre o número de espécies encontradas entre um 
período e o outro. As espécies registradas neste estudo compõem um grupo de organismos que podem ser preditivos de boa qualidade da água, respondendo também aos baixos teores de $\mathrm{pH}$ e às mudanças na concentração de nutrientes, provenientes da área de drenagem ou do lançamento pontual de fontes de poluição orgânica.

Os dois reservatórios apresentam características de ambientes oligotróficos com relação às concentrações de fósforo total. Entretanto, a reduzida cobertura vegetal e a presença de áreas de pastagem no entorno das represas evidenciam a necessidade de práticas para a conservação da qualidade da água destinada à captação para abastecimento público, sendo indicada a recuperação das matas ciliares às margens de ambos os mananciais.

\section{AGRADECIMENTOS}

À FUNADESP pelo apoio financeiro.

\section{REFERÊNCIAS}

Anagnostidis K. \& Komárek J. (1988): Modern approach to the classification system of the cyanophytes 3: Oscillatoriales. - Algol. Stud. 50/53: 327-472.

Amorim, A. L.; Ribeiro, M.M.R.; Braga, C.F.C. 2016. Conflitos em bacias hidrográficas compartilhadas: o caso da bacia do rio Piranhas-Açu/PB-RN. Revista Brasileira de Recursos Hídricos. 21 (1): 36-45.

Andrén, C.; Jarlman, A. 2008. Benthic diatoms as indicators of acidity in streams. Fundamental and Applied Limnology. Archiv für Hydrobiologie. 173 (3): 237-253.

Arias, A.R.L.; Buss, D.S.; Albuquerque, C.; Inácio, A.F.; Freire, M.M.; Egler, M.; Mugnai, R.; Baptista, D.F. 2007. Utilização de bioindicadores na avaliação de impacto e no monitoramento da contaminação de rios e córregos por agrotóxicos. Ciência e Saúde Coletiva. 12 (1): 61-72.

Baker, M.A.; Guzman, G.; Ostermiller, J.D. 2009. Differences in nitrate uptake among benthic algal assemblages in a mountain stream. Journal of the North American Benthological Society. 28 (1): 24-33.

Bellinger, E.G.; Sigee, D.C. 2010. Freshwater algae: identification and use as Bioindicators. WileyBlackwell, Chichester, UK, $271 \mathrm{p}$.

Berry, H.A.; Lembi, C.A. 2000. Effects of temperature and irradiance on the seasonal variation of a Spirogyra (Chlorophyta) population in a Midwestern Lake (U.S.A.). Journal of Phycology. 36 (5): 841-851.
Bicudo, D.C.; Necchi, O.J.R.; Chamixaes, C.B. 1995. Periphyton studies in Brazil: present status and perspectives. In: Tundisi, J.B.; Bicudo, C.E.M.; Matsumura Tundisi, T. (ed.). Limnology in Brazil. Academia Brasileira de Ciências e Sociedade Brasileira de Limnologia, Rio de Janeiro. 37-58pp.

Bicudo, M.E.C.; Menezes, M. 2006. Gêneros de algas de águas continentais do Brasil. $2^{a}$ Edição. Rima, São Carlos, 502 p.

Bourrelly, P.C. 1981. Les algues d'eau douce: initiation à la systématique. Les algues jaunes et brunes, Chrysophycées, Paheophycées, Xanthophycées et Diatomées. Éditions N. Boubée é Cie, Paris, v.2.

Bourrelly, P. 1985. Les algues déau douce: initiation à la systematique, 3: les algues bleueset rouges et brunes. Paris: Éditions N. Boubée. 606p

Buratti, F.M.; Manganelli, M.; Vichi, S.; Stefanelli, M.; Scardala, S.; Testai, E.; Funari, E. 2017. Cyanotoxins: producing organisms, occurrence, toxicity, mechanism of action and human health toxicological risk evaluation. Archives of Toxicology. 91 (3): 1049-1130.

Cabral, J.B.P; Santos, F.F.; Nogueira; P.F.; Braga, C.C. 2012. Análise espacial de sólidos em suspensão em reservatórios do estado de Goiás: estudo de caso de UHE Caçu e Barra dos Coqueiros. Revista Geonorte. 2 (4): 126-137.

Calijuri, M. C.; Alves, M. S. A.; Santos, A. C. A. 2006. Cianobactérias e cianotoxinas em águas continentais. Rima, São Carlos, 118p.

Cavati, B.; Fernandes, V. de O. 2008. Algas perifíticas em dois ambientes do baixo rio Doce (lagoa Juparanã e rio Pequeno - Linhares, Estado do Espirito Santo, Brasil): variação espacial e temporal. Acta Scientiarum Biological Sciences. 30 (4): 439-448.

Coesel, P.F.M. 2001. A method for quantifying conservation value in lenthic freshwater habitats using desmids as indicator organims. Biodiversity and Conservation 10 (2):177-187.

Coesel, P.F.M.; Krienitz, L. 2008. Diversity and geographic distribution of desmids and other coccoid green algae. Biodiversity and Conservation, 17 (2): 381-392.

CONAMA, № 357, DE 17 DE MARÇO DE 2005.

http://pnqa.ana.gov.br/Publicacao/RESOLUCAO_ CONAMA_n_357.pdf.Acesso em 02/10/2018.

Cordeiro, S.; Barbosa, J.E.L.; Lima-Filho, G.Q.; Barbosa, L.G. 2017.Periphytic algae dynamics in lentic ecosystems in the Brazilian semiarid. Braz. J. Biol. 77 (3): $495-505$.

Cunha, D.G.; Bottino, F.; Callijuri, M. C. 2010. Land use influence on eutrophicationrelated water variables: case study of tropical rivers with different degrees 
of anthropogenic interference. Acta Limnologica Brasiliensia. 22 (1):35-45.

Dodds, W.K. 2003. The role periphyton in Phosphorus retention in shallow freshwater aquatic systems. Journal of Phycology 39:840-849.

Dunck, B.; Nogueira, I.S.; Felisberto, S.A. 2013. Distribution of periphytic algae in wetlands (Palm swamps, Cerrado), Brazil. Brazilian Journal of Biology. 73 (2): 331-346.

Dzialowski, A.R.Smith, V.H.; Huggins, D.G.; Denoyelles, F.; Lim, N.C.; Baker D.S.; Beury, J.H. 2009. Development of predictive models for geosminrelated taste and odor in Kansas, USA, drinking water reservoirs. Water Res. 43(11): 2829-40.

Espíndola, E.L.G.; Matsumura-Tundisi, T.; Riletzler, A.C.; Tundisi, J.G. 2000. Spatial heterogeneity of the Tucuruí reservoir (State of Pará, Amazonia, Brazil) and the distribution of zooplanktonic species. Revista Brasileira Biologia. 60 (2): 179-194.

Esteves, F. A. 2011. Fundamentos de Limnologia. $3^{\circ}$ Edição. Interciência, Rio de Janeiro, 826p.

Felisberto, S.A.; Rodrigues, L. 2005. Periphytic community of reservoirs cascade in the Paranapanema river, Brazil. Acta Scientiarum, Biological Sciences. 27 (3): 215-223.

Felisberto, S.A.; Rodrigues, L; Santos, H.S. 2014. Taxonomical and ecological characteristics of the desmids placoderms in reservoir: analyzing the spatial and temporal distribution. Acta Limnologica Brasiliensia. 26 (4): 392-403.

Fernandes, V.O.; Cavati, B.; Oliveira, L. B.; Souza, B.D. 2009. Ecologia de cianobactérias: fatores promotores e conseqüências das florações. Oecologia Brasiliensis. 13 (2): 247-258.

Ferragut, C.; Bicudo, D.C. 2012. Effect of $\mathrm{N}$ and $P$ enrichment on periphytic algal community succession in a tropical oligotrophic reservoir. Limnology.13 (1): 131-141.

Fonseca, I.A.; Rodrigues, L. 2005. Comunidade de algas perifíticas em distintos ambientes da planície de inundação do alto rio Paraná. Acta Scientiarum, Biological Sciences. 27 (1): 21-28.

Fonseca, I.A.; Siqueira, N.S.; Rodrigues, L. 2009. Algas perifíticas a montante e a jusante do local de instalação de tanques-rede em tributários do reservatório de Rosana, Estado do Paraná, Brasil. Acta Scientiarum. Biological Sciences. 31(2): 135-141.

França, R.C.S.; Lopes, M.R.M.; Ferragut, C. 2011. Structural and successional variability of periphytic algal community in a Amazonian lake during the dry and rainy season (Rio Branco, Acre). Acta Amazonica. 41 (2): 257-266.
Franceschini, I.M.; Burliga, A.L.; Reviers, B. 2010. Algas uma abordagem Filogenética. Artmed, Porto Alegre, 332p.

Freitas, F.R.S.; Richetto, A. M.; Attayde, J. L. 2011. Cargas de fósforo total e material em suspensão em um reservatório do semi-árido brasileiro. Oecologia Australis. 15 (3): 655-665.

García, J.M., Sarmiento, L.F., Salvador, M., Porras, L.S. 2017. Uso de bioindicado-res para la evaluación de la calidad del agua en ríos: aplicación en ríos tropicales de alta montaña. Revisión corta. UGCiencia. 23: 47-62.

Gorman, M. W.; Zimmer, K.D.; Herwig, B. R.; Hanson, M. A.; Wright, R. G.; Vaughn, S. R.; Younk, J. A. 2014. Relative importance of phosphorus, fish biomass, andwatershed land use as drivers of phytoplankton abundance in shallow lakes. Science of the Total Environment. 466-467: 849-855.

Graham, J.L.; Loftin, K.A.; Meyer, M.T.; Ziegler, A.C. 2010. Cyanotoxin Mixtures and Taste-and-Odor Compounds in Cyanobacterial Blooms from the Midwestern United States. Environ. Sci. Technol. 44 (19): 7361-7368.

Kaiser, H.F. 1958. The varimax criterion for analytic rotation in factor analysis. Psychometrika. 23: 187-200.

Komárek, J., Anagnostidis, K. 1989.Modern Approach to the Classification System of Cyanophytes 4. Nostocales. Archiv für Hydrobiologie, 82, 247-345.

Komárek, J., Fott, B. 1983. Chlorophyceae (Grünalgen), Ordiniung: Chlorococcales. In: G. Huber-Pestalozzi, H. Heynig \& D. Mollenhauer (eds.). Das Phytoplankton des Süsswasser: systematik und biologie. E. Schweizerbart'sche Verlagsbuchlandlung, Stuttgart, pp. 1-1044

Krupek, R.A.; Branco, C.C.Z.; Peres, C.K. 2008. Variação sazonal de alguns parâmetros físicos e químicos em três rios pertencentes a uma bacia de drenagem na região centro-sul do Estado do Paraná, Sul do Brasil. Acta Scientiarum. Biological Sciences. 30 (4): 431-438.

Lamparelli, M.C. 2004. Graus de trofia em corpos d'agua do estado de São Paulo: avaliação dos métodos de monitoramento. $235 \mathrm{f}$. Tese de Doutorado. Universidade de São Paulo - USP. 235p.

Legendre, P.; Legendre, L. 1998. Numerical ecology. $2^{\mathrm{a}}$ Edição, Elsevier Science, $853 \mathrm{p}$.

Li, L.; Zheng, B.; Liu, L. 2010. Biomonitoring and bioindicators used for river ecosystems: Definitions, approaches and trends. Procedia Environmental Sciences. 2: 1510-1524.

Lobo, E. 2013. O perifíton como indicador da qualidade da água. In: Schwarzbold, A.; Burliga, A. L.; Torgan, 
L. C. (ed.) Ecologia do perifíton. RiMa, São Carlos, 205-233pp.

Masseret, E.; Amblard, C.; Bourdier, G.; Sargos, D. 2000. Effects of a waste stabilization lagoon discharge on bacterial and phytoplanktonic communities of a stream, Water Environment Research. 72 (3): 285-294.

Mccormick, P.V.; Stevenson, R.J. 1998. Periphyton as a tool for ecological assessment and management in the Florida Everglades. Journal of Phycology. 34 (5):726 - 733.

Miretzky, P.; Maidana, N.I.; Cireli, A.F. 2002. Stability of diatom composition in a variable lake environment: Lake Chascomús, Argentina. Limnology. 2 (3): 77-85.

Murakami, E.A; Rodrigues, L. 2009. Resposta das algas perifíticas às alterações de temperatura $e$ ao enriquecimento artificial de nutrientes em curto período de tempo. Acta Scientiarum. Biological Sciences. 31 (3): 273-284.

Newman, S.; Mccormick, P.V.; Backur, J.G. 2003. Phosphatase activity as an early warning indicator of wetland eutrophication: problems and prospects. Journal of Applied Phycology 15 (1):45-59.

Ngearnpat, N.; Peerapornpisal, Y. 2007. Application of desmid diversity in assessing the water quality of 12 freshwater resources in Thailand. Journal of Applied Phycology, 19 (6): 667-674.

Oliveira S.A.; Bicudo, C. E. M. 2017. Seasonal variation of limnological features and trophic state index of two oligotrophic reservoirs of southeast Brazil. Braz. J. Biol. 77 (2): 323-331.

Oliveira, D.E.; Ferragut, C.; Bicudo, D.C. 2010. Relationships between environmental factors, periphyton biomass and nutrient content in Garças Reservoir, a hypereutrophic tropical reservoir in southeastern Brazil. Lakes; Reservoirs: Research and Management. 15 (2): 129-137.

Olsen, B.K.; Chislock, M.F.; Wilson, A.E. 2016. Eutrophication mediates a common off-flavor compound, 2- methylisoborneol, in a drinking water reservoir. Water Research. 92 (1): 228-34.

Pandey, L.K.; Bergey, E.A.; Lyud, J.; Park, J.; Choi, S.; Lee, H.; Depuydt, S.; Oh, Y.; Lee, S.; Han, T. 2017. The use of diatoms in ecotoxicology and bioassessment: Insights, advances and challenges. 118 (1): 39-58.

Pellegrini, B.G.; Ferragut, C. 2012. Variação sazonal e sucessional da comunidade de algas perifíticas em substrato natural em um reservatório mesotrófico tropical. Acta Botanica Brasilica. 26 (4): 807-818.

Siegel, S. 1975. Estatística Não-Paramétrica para as ciências do comportamento. McGraw-Hill do Brasil, São Paulo, 350p.
Sládecková, A. 1991. The role of periphyton in water supply. Verhandlungen des Internationalen Verein Limnologie. 24 (4): 2174-2178.

Stamenković, M.; Hanelt, D. 2017.Geographic distribution and ecophysiological adaptations of desmids (Zygnematophyceae, Streptophyta) in relation to PAR, UV radiation and temperature: a review. Hydrobiologia. 787 (1): 1-26.

Št'astný, J. 2009. The desmids of the Swamp Nature Reserve (North Bohemia, Czech Republic) and a small neighbouring bog: species composition and ecological condition of both sites. Fottea. 9 (1): 135-148.

Straskraba, M.; Tundisi, J. G. 2000. Gerenciamento da qualidade da água de represas. In: Tundisi, J. G. (ed.) Diretrizes para o gerenciamento de lagos. ILEC/IEE, São Carlos. 87-102pp.

Sun, D.; Yu, Ji; Na, W.; Yang, M.; Chen, G.; Zhang, S. 2013. Identification of causative compounds and microorganisms for musty odor occurrence in the Huangpu River, China. Journal of Environmental Sciences 25 (3): 460-465.

Taniwaka, R.H.; Borguit, T.C.; Magrin A.G.E.; Calijuri, M.C.; Bottino, F.; Moschini-Carlos, V. 2013. Structure and dynamics of the community of periphytic algae in a subtropical reservoir (state of São Paulo, Brazil). Acta Bot Bras. 27 (3): 551-559.

Tundisi, J. G.; Matsumura-Tundisi; Pareschi, D.C.; Luzia, A.P.; Von Haeling, P.H.; Frollini, E.H. 2008. A bacia hidrográfica do Tietê/Jacaré: estudo de caso em pesquisa e gerenciamento. Estudos Avançados. 22 (63): 159-172.

Tundisi, J.G. 2003. Água no século XXI enfrentando a escassez. RiMa/iiE, São Carlos, 248p.

Tundisi, J.G.; Tundisi, T.M. 2008. Limnologia. Oficina de Textos, São Paulo, 632p.

Vadeboncoeur, Y.; Steinman, A.D. 2002. Periphyton Function in Lake Ecosystems. The Scientific World Journal. 2: 1-20.

Valderrama, J.C. 1981. The simultaneous analysis of total nitrogen and phosphorus in natural waters. Marine Chemistry. 10: 1109-1122.

Vercellino, I.S.V.; Bicudo, D.C. 2006.Sucessão da comunidade de algas perifíticas em reservatório oligotrófico tropical (São Paulo, Brasil): comparação entre período seco e chuvoso. Revista Brasil. Bot. 29 (3): 363-377.

Vigil, K.M. 2003. Clean water: an introduction to water quality and water pollution control. Corvallis, Oregon State University Press. $181 \mathrm{p}$.

Winter, J.G.; Duthie, H.C. 2000. Epilithic diatoms as indicators of stream total $\mathrm{N}$ and $\mathrm{P}$ concentration. Journal of the North American Benthological Society. 19 (1): 32-49. 
Wu, N.; Tang, T.; Zhou, S.; Jia, X.; Li, D.; Liu, R.; Cai, Q. 2009. Changes in benthic communities following construction of run-of-river dam. Journal of the North American Benthological Society. 28 (1): 69-79.

Wunsam, S.; Cattaneo, A.; Bourassa, N. 2002. Comparing diatom species, genera and size in biomonitoring: a case study from streams in the Laurentians (Quebec, Canada). Freshwater Biology. 47 (2): 325-340.and Management, Ralph Wurbs (Ed.), London: InTech, p. 29-51.Horizonte: Universidade Federal de Minas Gerais (UFMG), $470 \mathrm{p}$.

Submetido: Maio18

Revisado: Setembro/18 Aceito:Outubro/18

Publicado: Abril/19 